\title{
On negative Pell equations: Solvability and unsolvability in integers
}

\section{Hsin-Te Chiang ${ }^{1}$, Mei-Ru Ciou ${ }^{2}$, Chia-Ling Tsai ${ }^{3}$, Yuh-Jenn $\mathrm{Wu}^{4}$ and Chiun-Chang Lee ${ }^{5}$}

${ }^{1}$ Institute for Computational and Modeling Science, National Tsing Hua University Hsinchu 30014, Taiwan e-mail: u10210214@mail.nd.nthu.edu.tw

${ }^{2}$ Institute for Computational and Modeling Science, National Tsing Hua University Hsinchu 30014, Taiwan e-mail: g10524201@mail.nd.nthu. edu.tw

${ }^{3}$ Institute for Computational and Modeling Science, National Tsing Hua University Hsinchu 30014, Taiwan e-mail: g10524214@mail.nd.nthu. edu.tw

${ }^{4}$ Department of Applied Mathematics, Chung Yuan Christian University

Taoyuan City 32023, Taiwan e-mail: yuh jenn@cycu.edu.tw

${ }^{5}$ Institute for Computational and Modeling Science, National Tsing Hua University Hsinchu 30014, Taiwan e-mail: chlee@mail.nd.nthu.edu.tw

Received: 5 October 2017

Accepted: 28 September 2018

Abstract: Solvability criteria of negative Pell equations $x^{2}-d y^{2}=-1$ have previously been established via calculating the length for the period of the simple continued fraction of $\sqrt{d}$ and checking the existence of a primitive Pythagorean triple for $d$. However, when $d \gg 1$, such criteria usually require a lengthy calculation. In this note, we establish a novel approach to construct integers $d$ such that $x^{2}-d y^{2}=-1$ is solvable in integers $x$ and $y$, where $d=d\left(u_{n}, u_{n+1}, m\right)$ can be expressed as rational functions of $u_{n}$ and $u_{n+1}$ and fourth-degree polynomials of $m$, and $u_{n}$ satisfies a recurrence relation: $u_{0}=u_{1}=1$ and $u_{n+2}=3 u_{n+1}-u_{n}$ for $n \in \mathbb{N} \cup\{0\}$. Our main 
argument is based on a binary quadratic relation between $u_{n}$ and $u_{n+1}$ and properties $\frac{1+u_{n}^{2}}{u_{n+1}} \in \mathbb{N}$ and $\frac{1+u_{n+1}^{2}}{u_{n}} \in \mathbb{N}$. Due to the recurrence relation of $u_{n}$, such $d$ 's are easy to be generated by hand calculation and computational mathematics via a class of explicit formulas. Besides, we consider equation $x^{2}-k(k+4) m^{2} y^{2}=-1$ and show that it is solvable in integers if and only if $k=1$ and $m \in \mathbb{N}$ is a divisor of $\frac{1}{2} u_{3 n+2}$ for some $n \in \mathbb{N} \cup\{0\}$. The main approach for its solvability is the Fermat's method of infinite descent.

Keywords: Negative Pell equations, Quadratic Diophantine equations, Fermat's method of infinite descent.

AMS Classification: 11D45, 11D25.

\section{Introduction and the statement of main results}

This work is devoted to constructing a class of positive integers $d \equiv d\left(u_{n}, u_{n+1}, m\right)$ expressed by $u_{n}, u_{n+1}$ and fourth-degree polynomials of $m$ such that the negative Pell equation $x^{2}-d y^{2}=-1$ is solvable in integers, where $\left\{u_{n}\right\}_{n \in \mathbb{N} \cup\{0\}}$ satisfies $u_{0}=u_{1}=1$ and $u_{n+2}=3 u_{n+1}-u_{n}$ for $n \in \mathbb{N} \cup\{0\}$. On the other hand, due to related works $[4,5]$, we are also interested in a special equation $x^{2}-k(k+4) m^{2} y^{2}=-1$, where $k, m \in \mathbb{N}$. A sufficient and necessary condition for the solvability of this equation in integers has been established. Before introducing the main ideas and the results, we recall the history of the Pell equation and some related works.

A well-known problem on finding nontrivial integer solutions of the Pell equation

$$
x^{2}-d y^{2}=c
$$

has a history of several hundred years (cf. $[8,11]$ ), where $c \in \mathbb{Z}-\{0\}$ and $d$ is usually assumed a positive square-free integer. Equation (1.1), a special case of binary quadratic Diophantine equations $A_{11} x^{2}+A_{12} x y+A_{22} y^{2}+B_{1} x+B_{2} y=c$ with $A_{11} A_{22} \neq 0$ and $A_{12}^{2}-4 A_{11} A_{22}>0$, was named after the mathematician John Pell. A nature of (1.1) is that if it has an integer solution $(x, y)$ with $x y \neq 0$, then it must have infinitely many distinct integer solutions. Indeed, it is known (proved by Lagrange in 1768) that $x^{2}-d y^{2}=1$ has nontrivial integer solutions for all square-free integer $d \geq 2$, and its all integer solutions can be generated by its fundamental solution $(x, y)=\left(x_{0}, y_{0}\right)$ (which means $x_{0}, y_{0} \in \mathbb{N}$ and $x_{0}+y_{0}=\min \{x+y: x, y \in$ $\mathbb{N}$ and $(x, y)$ solves $(1.1)\})$. Assume that $(x, y)=\left(a_{0}, b_{0}\right)$ is an integer solution of (1.1). Then one may check that $(x, y)=\left(a_{n}, b_{n}\right)$ also solves $(1.1)$, where $n \in \mathbb{N}$ and

$$
\begin{aligned}
& a_{n}=\frac{1}{2}\left[\left(a_{0}+\sqrt{d} b_{0}\right)\left(x_{0}+\sqrt{d} y_{0}\right)^{n-1}+\left(a_{0}-\sqrt{d} b_{0}\right)\left(x_{0}-\sqrt{d} y_{0}\right)^{n-1}\right] \in \mathbb{Z}, \\
& b_{n}=\frac{1}{2 \sqrt{d}}\left[\left(a_{0}+\sqrt{d} b_{0}\right)\left(x_{0}+\sqrt{d} y_{0}\right)^{n-1}-\left(a_{0}-\sqrt{d} b_{0}\right)\left(x_{0}-\sqrt{d} y_{0}\right)^{n-1}\right] \in \mathbb{Z} .
\end{aligned}
$$

These solutions can be obtained directly from the Binomial theorem and establishing the recurrence relations. It is also known that even if (1.1) is solvable in integers $x$ and $y$, finding its fundamental solution may not be an easy matter. A famous example is equation $x^{2}-991 y^{2}=1$. 
Its fundamental solution given as follows is quite huge (cf. [10]; see also, the online solver [1]):

$$
(x, y)=(379516400906811930638014896080,12055735790331359447442538767) \text {. }
$$

On the other hand, (1.1) may be unsolvable for some $d$ and $c$.

For instance, $x^{2}-2 y^{2}=3$ is unsolvable in integers $x$ and $y$, which can be immediately proved by applying congruence modulo 3 to this equation. Hence, when $c \neq 1$, the situation for solvability of (1.1) becomes more complicated. A quite interesting question immediately arises:

"Given $c \neq 1$, for what value of $d,(1.1)$ is solvable in integers $x$ and $y ?$ ?"

For this question, a crucial case is $c=-1$; that is the negative Pell equation (cf. [3])

$$
x^{2}-d y^{2}=-1(x, y \in \mathbb{Z}) .
$$

A necessary condition for solvability of (1.2) in integers is that $d \equiv 1$ or $2(\bmod 4)$ and all odd prime divisors of $d$ are of the forms congruent to 1 modulo 4 . However, these conditions are not sufficient for a solution to exist. One may visit A031396 in the OEIS [9] for some known numbers $d=2,5,10,13,17,26,29,37,41,50, \ldots$ such that (1.2) is solvable in integers $x$ and $y$. Within decades, some number theorists are devoted to establishing criteria for solvability of (1.2). Some criteria can be found in [2, 6, 7, 10]. Newman [7] showed that if $d=\prod_{i=1}^{r} p_{i}$, where $r=2$ or $r$ is odd, and $p_{i}$ 's are primes congruent to 1 modulo 4 and satisfy $\left(\frac{p_{i}}{p_{j}}\right)=-1$ for all $1 \leq i, j \leq r$ with $i \neq j$, then (1.2) is solvable in integers. Here $(\vdots)$ is the usual Jacobi symbol. On the other hand, Mollin [6] established a connection between the negative Pell equation (1.2) and the equation $x^{2}-d y^{2}=1$. He showed that (1.2) is solvable in integers $x$ and $y$ if and only if the fundamental solution $\left(x_{0}, y_{0}\right)$ of $x^{2}-d y^{2}=1$ satisfies $x_{0} \equiv-1(\bmod 2 d)$. Although these results are quite important, their methods are limited if $d \gg 1$. For example, there are many cases that their fundamental solutions are quite large. Hence, checking these conditions needs lengthy calculations. Recently, well-known solvability criteria of negative Pell equations have been established. One method is to calculate the length for the period of the simple continued fraction of $\sqrt{d}$; the other one is to check the existence of a primitive Pythagorean triple for $d$. We state these two criteria as follows:

Theorem A1 (cf. [10] in 1964). Let $\sqrt{d}=\left[q_{0} ; \overline{q_{1}, \ldots, q_{s}}\right]$ be the representation of $\sqrt{d}$ as a simple continued fraction with period s, then (1.2) is solvable in integers $x$ and $y$ if and only if the period $s$ is odd.

Theorem A2 (cf. [2] in 2000). (1.2) is solvable in integers $x$ and $y$ if and only if there exist a primitive Pythagorean triple $(A, B, C)$ and positive integers $\alpha$ and $\beta$ such that

$$
d=\alpha^{2}+\beta^{2} \quad \text { and } \quad|A \alpha-B \beta|=1 .
$$

Here $(A, B, C)$ is said to be a primitive Pythagorean triple if they are positive integers satisfying $A^{2}+B^{2}=C^{2}$ with $A$ and $B$ relatively prime. Moreover, $(x, y)=(B \alpha+A \beta, C)$ gives a solution of (1.2). 
Hence, to verify the solvability for (1.2), one can check the period length for the simple continued fraction of $\sqrt{d}$ or check the existence of a primitive Pythagorean triple for $d$. However, when $d \gg 1$, such criteria usually require a lengthy calculation. This motivates us to develop a new method for constructing explicit forms of $d$ such that (1.2) is solvable in integers. For convenience of our argument, we only consider the case that $d$ is odd and all of its prime divisor are congruent to 1 modulo 4 . (Note that $d$ can be not a square-free number.) Our main idea is to consider a factor decomposition $d=a b$ for (1.2), i.e.,

$$
x^{2}-a b y^{2}=-1, \quad \text { for } x, y \in \mathbb{Z},
$$

and transform (1.4) into the quadratic Diophantine equation

$$
X^{2}-(a+b) X Y+\left(\frac{a-b}{2} Y\right)^{2}+1=0
$$

under the relations

$$
x=X-\frac{a+b}{2} Y, \quad y=Y .
$$

Note that when $a=b>1,(1.4)$ is unsolvable in the integers. Note also that $a \equiv b \equiv 1(\bmod 4)$. Without loss of generality, we may assume $1 \leq a<b$ satisfying

$$
b=a+4 m, \quad m \in \mathbb{N} .
$$

Accordingly, $(X, Y)=\left(X_{0}, Y_{0}\right)$ is an integer solution of (1.5) if and only if $(x, y)$ $=\left( \pm\left(X_{0}-\frac{a+b}{2} Y_{0}\right), \pm Y_{0}\right)$ is an integer solution of (1.4). Moreover, by (1.7), one immediately finds that all prime divisors of $X_{0}$ and $Y_{0}$ are congruent to 1 modulo 4 . Next, we attempt to birth a breakthrough idea to connect a second-order linear homogeneous recurrence sequence $A u_{n+2}=$ $B u_{n+1}+C u_{n}$ and the equation (1.4). Such an intuition comes from a fact that $u_{n}$ satisfies a quadratic form $C u_{n}^{2}+B u_{n} u_{n+1}-A u_{n+1}^{2}=A\left(u_{n} u_{n+2}-u_{n+1}^{2}\right)=\left(-\frac{C}{A}\right)^{n}\left(C u_{0}^{2}+B u_{0} u_{1}-A u_{1}^{2}\right)$ (see the Appendix (Section 3.3)), which has similar form as (1.5) when $A=1$ and $C=-1$. This gives a connection to constructing a new class of $d=a b$ such that (1.4) is solvable. Although the quadratic form of $u_{n}$ and $u_{n+1}$ is well-known, to the best of our knowledge, it seems that rare literature gives a connection to construct a wide variety of $d=a b$ such that (1.4) is solvable. Using such an idea, we can use some $u_{n}$ 's to generate a rich class of values $a$ and $b$ such that (1.4) is solvable in integers $x$ and $y$. Due to the recurrence relation of $u_{n}$, these $d=a b$ are easy to be generated by hand calculation and computational mathematics via a class of explicit formulas. In Section 3, we will provide some examples (see also, Tables 1-4) by hand computation, which come from the following main result.

Theorem 1.1. Define a sequence $\left\{u_{n}\right\}_{n \in \mathbb{N} \cup\{0\}}$ satisfying

$$
u_{0}=u_{1}=1, \quad u_{n+2}=3 u_{n+1}-u_{n}, n \in \mathbb{N} \cup\{0\} .
$$

In addition, for the convenience of notations, we define $u_{-1}:=2$ and $u_{-2}:=5$. Then (1.4) is solvable in integers $x$ and $y$ when $a$ and $b$ satisfy one of the following cases: 
(i) For each $m \in \mathbb{N}$ satisfying $m^{2} \equiv 1\left(\bmod u_{3 n+1}\right)$, we choose $(a, b)=\left(a_{n}(m), b_{n}(m)\right)$, where

$$
a_{n}(m)=\frac{\left(m^{2}-1\right) u_{3 n+2}}{u_{3 n+1}}+3-2 m \quad \text { and } \quad b_{n}(m)=\frac{\left(m^{2}-1\right) u_{3 n+2}}{u_{3 n+1}}+3+2 m .
$$

Then (1.4) has an integer solution

$$
\left\{\begin{array}{l}
x=\frac{\left(m^{2}-1\right) u_{3 n+2}^{2}}{2 u_{3 n+1}}+\frac{u_{3 n+3}-u_{3 n+1}}{2} \\
y=\frac{1}{2} u_{3 n+2}
\end{array}\right.
$$

(ii) For each $m \in \mathbb{N}$ satisfying $m^{2} \equiv 1\left(\bmod u_{3 n}\right)$, we choose $(a, b)=\left(a_{n}(m), b_{n}(m)\right)$, where

$$
a_{n}(m)=\frac{\left(m^{2}-1\right) u_{3 n-1}}{u_{3 n}}+3-2 m \quad \text { and } \quad b_{n}(m)=\frac{\left(m^{2}-1\right) u_{3 n-1}}{u_{3 n}}+3+2 m .
$$

Then (1.4) has an integer solution

$$
\left\{\begin{array}{l}
x=\frac{\left(m^{2}-1\right) u_{3 n-1}^{2}}{2 u_{3 n}}-\frac{u_{3 n}-u_{3 n-2}}{2} \\
y=\frac{1}{2} u_{3 n-1}
\end{array}\right.
$$

(iii) For each $m \in \mathbb{N}$ satisfying $4 m^{2} \equiv 1\left(\bmod u_{3 n}\right)$, we choose $(a, b)=\left(a_{n}(m), b_{n}(m)\right)$, where $a_{n}(m)=\frac{\left(4 m^{2}-1\right) u_{3 n+1}+3 u_{3 n}}{2 u_{3 n}}-2 m \quad$ and $\quad b_{n}(m)=\frac{\left(4 m^{2}-1\right) u_{3 n+1}+3 u_{3 n}}{2 u_{3 n}}+2 m$.

Then (1.4) has an integer solution

$$
\left\{\begin{array}{l}
x=\frac{\left(4 m^{2}-1\right) u_{3 n+1}^{2}-u_{3 n}^{2}}{2 u_{3 n}}+\frac{1}{2} u_{3 n+2} \\
y=u_{3 n+1}
\end{array}\right.
$$

(iv) For each $m \in \mathbb{N}$ satisfying $4 m^{2} \equiv 1\left(\bmod u_{3 n+1}\right)$, we choose $(a, b)=\left(a_{n}(m), b_{n}(m)\right)$, where

$a_{n}(m)=\frac{\left(4 m^{2}-1\right) u_{3 n}+3 u_{3 n+1}}{2 u_{3 n+1}}-2 m \quad$ and $\quad b_{n}(m)=\frac{\left(4 m^{2}-1\right) u_{3 n}+3 u_{3 n+1}}{2 u_{3 n+1}}+2 m$.

Then (1.4) has an integer solution

$$
\left\{\begin{array}{l}
x=\frac{\left(4 m^{2}-1\right) u_{3 n}^{2}-u_{3 n+1}^{2}}{2 u_{3 n+1}}+\frac{1}{2} u_{3 n-1}, \\
y=u_{3 n} .
\end{array}\right.
$$

We shall state the proof of Theorem 1.1 in Section 2.1. As an application of Theorem 1.1, we refer the reader to Examples 1-3 in Section 3. 
Remark 1. In Theorem 1.1, $d=a b$ is of a fourth-degree polynomial of $m$. Such expressions of $d$ 's are new, and do not appear in the literatures related to the polynomial Pell equations.

In $[4,5]$, the authors ever studied the solvability in integers $x$ and $y$ for the following Pell equation $x^{2}-k(k+4) y^{2}=-4$ by using an infinite simple continued fraction of $\sqrt{k(k+4)}$, where $k \in \mathbb{N}$. As a special case of (1.4) with $a=k m$ and $b=(k+4) m$, we use a different approach to establish a sufficient and necessary condition for the solvability of

$$
x^{2}-k(k+4) m^{2} y^{2}=-1
$$

in integers. We stress that (1.17) includes $x^{2}-k(k+4) y^{2}=-4$ in the case that $m$ is even. Moreover, using Theorem A1, we obtain the period length for the simple continued fraction of $\sqrt{k(k+4)} m$. Such results are stated as follows:

Theorem 1.2. Let $k, m \in \mathbb{N}$. Then

(i) When $k \geq 2$, for any $m \in \mathbb{N}$, (1.17) is unsolvable in integers. In particular, this implies that for $k \geq 2$, the period of the simple continued fraction of $m \sqrt{k(k+4)}$ is even.

(ii) When $k=1,(1.17)$ is solvable in integers if and only if $m$ is a divisor of $\frac{1}{2} u_{3 n+2}$ for some $n \in \mathbb{N} \cup\{0\}$. In particular, the period of the simple continued fraction of $\sqrt{5} \mathrm{~m}$ is even if and only if

$$
m \nmid \frac{1}{2} u_{3 n+2}, \quad \forall n \in \mathbb{N} \cup\{0\},
$$

where $u_{n}$ is defined in (1.8).

The main approach for the unsolvability comes from the concept of the Fermat's method of infinite descent. The proof of Theorem 1.2 will be given in Section 2.2.

Besides, we also provide Examples $4-5$ for the solvability and unsolvability of (1.17). In particular, using Theorem 1.2(ii) we show that $x^{2}-5 \cdot 37^{2} y^{2}=-1$ is solvable in integers, while $x^{2}-5 \cdot 29^{2} y^{2}=-1$ and $x^{2}-5 \cdot 41^{2} y^{2}=-1$ are unsolvable in integers (cf. Example 5).

\section{Proof of main results}

\subsection{Proof of Theorem 1.1}

To prove Theorem 1.1, we need the following lemma:

Lemma 2.1. The sequence $\left\{u_{n}\right\}_{n \in \mathbb{N}}$ presented in (1.8) has the following properties.

(i) $u_{n}^{2}-3 u_{n} u_{n+1}+u_{n+1}^{2}+1=0, n \in \mathbb{N} \cup\{0\}$.

(ii) For each $n \in \mathbb{N} \cup\{0\}$, all odd prime divisors $p$ of $u_{n}$ satisfy $p \equiv 1$ ( $\left.\bmod 4\right)$. Moreover, $u_{3 n} \equiv u_{3 n+1} \equiv 1(\bmod 4)$, and $u_{3 n+2} \equiv 2(\bmod 4)$. 
Proof. It is apparent that $u_{n} \in \mathbb{N}$ and

$$
u_{n+2} \equiv-\left(u_{n+1}+u_{n}\right)(\bmod 4) .
$$

On the other hand, by (1.8) and Proposition 3.1, we immediately get (i) and $u_{n} u_{n+2}=u_{n+1}^{2}+1$, $\forall n \in \mathbb{N} \cup\{0\}$. In particular, one finds that $u_{n}$ and $u_{n+1}$ are relatively prime and satisfy

$$
u_{n} \mid\left(u_{n+1}^{2}+1\right) \quad \text { and } u_{n+1} \mid\left(u_{n}^{2}+1\right), \quad \forall n \in \mathbb{N} \cup\{0\} .
$$

Let $p \geq 3$ be a prime divisor of $u_{n}$. By (2.2) we have

$$
u_{n+1}^{4} \equiv 1(\bmod p), u_{n+1}^{2} \equiv-1(\bmod p) .
$$

Along with the Fermat's little theorem gives $4 \mid(p-1)$. As a consequence, all possible prime divisors $p$ of $u_{n}$ are $p=2$ and $p \equiv 1(\bmod 4)$. Furthermore, we have that:

$$
\text { if } u_{n} \text { is odd, then } u_{n} \equiv 1(\bmod 4) \text {. }
$$

Using (2.1) and (2.3) and applying the mathematical induction, we get $u_{3 n}, u_{3 n+1} \equiv 1(\bmod 4)$ and $u_{3 n+2} \equiv 2(\bmod 4)$. Therefore, we prove (ii) and complete the proof of Lemma 2.1.

Now we state the proof of Theorem 1.1.

By (1.5) and (1.7), we have

$$
X^{2}-2(a+2 m) X Y+4 m^{2} Y^{2}+1=0 .
$$

Assume it has an integer solution $\left(X_{0}, Y_{0}\right)$. Then, $X_{0} \mid\left(4 m^{2} Y_{0}^{2}+1\right)$. We consider two cases as follows:

- Case 1. Assume $m^{2} \equiv 1\left(\bmod X_{0}\right)$. Then we can let $m^{2}=K X_{0}+1$ for some $K \in \mathbb{Z}$. By (2.4), we have

$$
X_{0}^{2}-\left(a+2 m-2 K Y_{0}\right) X_{0}\left(2 Y_{0}\right)+\left(2 Y_{0}\right)^{2}+1=0 .
$$

Hence, by Lemma 2.1 and (2.5), we can choose

$$
\left(X_{0}, Y_{0}, a+2 m-2 K Y_{0}\right)=\left(u_{3 n+1}, \frac{1}{2} u_{3 n+2}, 3\right) \text { or }\left(u_{3 n+3}, \frac{1}{2} u_{3 n+2}, 3\right) .
$$

By (1.7) and (2.6), we have

$$
a=2 K Y_{0}+3-2 m=\frac{2\left(m^{2}-1\right) Y_{0}}{X_{0}}+3-2 m, \quad b=\frac{2\left(m^{2}-1\right) Y_{0}}{X_{0}}+3+2 m .
$$

(A). If $\left(X_{0}, Y_{0}, a+2 m-2 K Y_{0}\right)=\left(u_{3 n+1}, \frac{1}{2} u_{3 n+2}, 3\right)$, then we have $m^{2} \equiv 1\left(\bmod u_{3 n+1}\right)$ and (1.9). Moreover, by (1.6) and (2.7) we obtain

$$
\begin{aligned}
x_{0} & :=X_{0}-\frac{a+b}{2} Y_{0}=u_{3 n+1}-\left(\frac{\left(m^{2}-1\right) u_{3 n+2}}{u_{3 n+1}}+3\right) \frac{1}{2} u_{3 n+2} \\
& =\frac{2 u_{3 n+1}^{2}-\left(m^{2}-1\right) u_{3 n+2}^{2}-3 u_{3 n+1} u_{3 n+2}}{2 u_{3 n+1}} \\
& =\frac{u_{3 n+1}^{2}-\left(m^{2}-1\right) u_{3 n+2}^{2}+u_{3 n+1}\left(u_{3 n+1}-3 u_{3 n+2}\right)}{2 u_{3 n+1}} \\
& =-\frac{\left(m^{2}-1\right) u_{3 n+2}^{2}}{2 u_{3 n+1}}+\frac{u_{3 n+1}-u_{3 n+3}}{2}, \\
y_{0} & :=Y_{0}=\frac{1}{2} u_{3 n+2} .
\end{aligned}
$$


Choosing $(x, y)=\left(-x_{0}, y_{0}\right)$, we get (1.10) an integer solution of (1.4). Therefore, we complete the proof of (i).

(B). If $\left(X_{0}, Y_{0}, a+2 m-2 K Y_{0}\right)=\left(u_{3 n+3}, \frac{1}{2} u_{3 n+2}, 3\right)$, following the similar argument of (A), we can prove (ii).

- Case 2. Assume $4 m^{2} \equiv 1\left(\bmod X_{0}\right)$. Then we can let $4 m^{2}=K X_{0}+1$ for some $K \in \mathbb{Z}$. By (2.4), we have

$$
X_{0}^{2}-\left(2 a+4 m-K Y_{0}\right) X_{0} Y_{0}+Y_{0}^{2}+1=0 .
$$

Note that $X_{0}$ must be odd. On the other hand, comparing Lemma 2.1(i) and (2.8), we shall choose

$$
2 a+4 m-K Y_{0}=3 .
$$

Hence, $Y_{0}$ is also odd and

$$
a=\frac{\left(4 m^{2}-1\right) Y_{0}+3 X_{0}}{2 X_{0}}-2 m \quad \text { and } \quad b=\frac{\left(4 m^{2}-1\right) Y_{0}+3 X_{0}}{2 X_{0}}+2 m .
$$

Consequently, by Lemma 2.1(ii) and (2.8), we can choose

$$
\left(X_{0}, Y_{0}\right)=\left(u_{3 n}, u_{3 n+1}\right) \text { or }\left(u_{3 n+1}, u_{3 n}\right)
$$

(C). If $\left(X_{0}, Y_{0}\right)=\left(u_{3 n}, u_{3 n+1}\right)$, then $4 m^{2} \equiv 1\left(\bmod u_{3 n}\right)$. By (2.9) and (2.10) we get $(1.13)$, and

$$
\begin{aligned}
x_{0} & :=X_{0}-\frac{a+b}{2} Y_{0}=u_{3 n}-\frac{\left(4 m^{2}-1\right) u_{3 n+1}^{2}+3 u_{3 n} u_{3 n+1}}{2 u_{3 n}} \\
& =\frac{2 u_{3 n}^{2}-\left(4 m^{2}-1\right) u_{3 n+1}^{2}-3 u_{3 n} u_{3 n+1}}{2 u_{3 n}} \\
& =\frac{u_{3 n}^{2}-\left(4 m^{2}-1\right) u_{3 n+1}^{2}+u_{3 n}\left(u_{3 n}-3 u_{3 n+1}\right)}{2 u_{3 n}} \\
& =\frac{u_{3 n}^{2}-\left(4 m^{2}-1\right) u_{3 n+1}^{2}}{2 u_{3 n}}-\frac{1}{2} u_{3 n+2}, \\
y_{0}: & =Y_{0}=u_{3 n+1} .
\end{aligned}
$$

Choosing $(x, y)=\left(-x_{0}, y_{0}\right)$, we get (1.14) an integer solution of (1.4). Therefore, we complete the proof of (iii).

(D). If $\left(X_{0}, Y_{0}\right)=\left(u_{3 n+1}, u_{3 n}\right)$, then $4 m^{2} \equiv 1\left(\bmod u_{3 n+1}\right)$. Following the similar argument as $(\mathrm{C})$, we can prove (iv).

Therefore, we complete the proof of Theorem 1.1. 


\subsection{Proof of Theorem 1.2}

We will prove Theorem 1.2(i) by contradiction. One may check that (1.17) is solvable in integers if and only if

$$
X^{2}-2(k+2) m X Y+4 m^{2} Y^{2}+1=0
$$

is solvable in integers (by (1.5) and (1.6)). Suppose that there exist $k \geq 2$ and $m \in \mathbb{N}$ such that (2.11) has an integer solution $\left(X_{0}, Y_{0}\right)$. Let $Z=2 m Y$. Then

$$
X^{2}-(k+2) X Z+Z^{2}+1=0
$$

has an integer solution $\left(X_{0}, Z_{0}\right):=\left(X_{0}, 2 m Y_{0}\right)$ with $X_{0} Z_{0}>0$ and $\left(-X_{0},-Z_{0}\right)$ also solves (2.12). Hence, without loss of generality, we may assume that (2.12) has an integer solution $(X, Z)=\left(\widehat{X}_{0}, \widehat{Z}_{0}\right)$, where $\widehat{X}_{0}, \widehat{Z}_{0} \in \mathbb{N}$ satisfy

$$
\widehat{X}_{0}+\widehat{Z}_{0}=\min \{X+Z: X, Z \in \mathbb{N} \text { and }(X, Z) \text { solves (2.12). }\} .
$$

(2.13) is well-defined due to the well-ordering property for the natural numbers. Moreover, by (2.12), we have

$$
\frac{\widehat{Z}_{0}^{2}+1}{\widehat{X}_{0}} \in \mathbb{N} \quad \text { and } \quad \frac{\widehat{X}_{0}^{2}+1}{\widehat{Z}_{0}} \in \mathbb{N} .
$$

We consider two cases as follows:

- Case 1. If $\widehat{Z}_{0}<\widehat{X}_{0}$, then putting $Z=\widehat{Z}_{0}$ in (2.12) and applying the Vieta's formulas, one finds that $(X, Z)=\left(\widehat{X}_{1}, \widehat{Z}_{0}\right)$ is another integer solution, where $\widehat{X}_{1}=(k+2) \widehat{Z}_{0}-\widehat{X}_{0}=$ $\frac{\widehat{Z}_{0}^{2}+1}{\widehat{X}_{0}} \in \mathbb{N}$ (by (2.14)). Note that $\widehat{Z}_{0} \geq 2$. We have

$$
1 \leq \widehat{X}_{1}=\frac{\widehat{Z}_{0}^{2}+1}{\widehat{X}_{0}} \leq \frac{\widehat{Z}_{0}^{2}+1}{\widehat{Z}_{0}+1}<\widehat{Z}_{0}<\widehat{X}_{0} .
$$

However, this implies $\widehat{X}_{1}+\widehat{Z}_{0}<\widehat{X}_{0}+\widehat{Z}_{0}$ which gives a contradiction to (2.13).

- Case 2. If $\widehat{X}_{0}<\widehat{Z}_{0}$, then by the Vieta's formulas and (2.14) one finds that (2.12) also has an other integer solutions $(X, Z)=\left(\widehat{X}_{0}, \widehat{Z}_{1}\right)$, where

$$
\widehat{Z}_{1}=(k+2) \widehat{X}_{0}-\widehat{Z}_{0}=\frac{\widehat{X}_{0}^{2}+1}{\widehat{Z}_{0}} \leq \frac{\widehat{X}_{0}^{2}+1}{\widehat{X}_{0}+1} \leq \widehat{X}_{0}<\widehat{Z}_{0} .
$$

As a result, we have $\widehat{X}_{0}+\widehat{Z}_{1}<\widehat{X}_{0}+\widehat{Z}_{0}$ which contradicts to (2.13).

Hence, by Cases 1 and 2, we have $\widehat{X}_{0}=\widehat{Z}_{0}$. Along with (2.12), we immediately get $k \widehat{X}_{0}^{2}=1$, which implies $k=\widehat{X}_{0}=1$, a contradiction. Consequently, we prove that for any $k \geq 2$ and $m \in \mathbb{N},(2.11)$ is unsolvable in integers. Moreover, by Theorem A1, we obtain that the period of the simple continued fraction of $m \sqrt{k(k+4)}$ is even for any $k \geq 2$ and $m \in \mathbb{N}$. Therefore, we complete the proof of (i).

It remains to prove (ii). When $k=1,(1.17)$ becomes

$$
x^{2}-5 m^{2} y^{2}=-1 \text {. }
$$

We need the following: 
Lemma 2.2. All positive integer solutions of $T^{2}-5 S^{2}=-1$ are of the form $\left(T_{n}, S_{n}\right)$, where

$$
T_{n}=\frac{3}{2} u_{3 n+2}-u_{3 n+1}, \quad S_{n}=\frac{1}{2} u_{3 n+2}, n \in \mathbb{N} \cup\{0\} .
$$

Proof. Using the fundamental solution $\left(T_{0}, S_{0}\right)=(2,1)$, one finds all positive integer solutions $\left(T_{n}, S_{n}\right)$ obeying

$$
T_{n}+S_{n} \sqrt{5}=(2+\sqrt{5})^{2 n+1}, \quad n \in \mathbb{N} \cup\{0\} .
$$

One may check from (2.19) that

$$
\begin{gathered}
T_{n}=\frac{1}{4}\left(S_{n+1}-9 S_{n}\right) \\
S_{0}=1, S_{1}=17, S_{n+2}=18 S_{n+1}-S_{n}, n \in \mathbb{N} \cup\{0\} .
\end{gathered}
$$

On the other hand, by applying the mathematical induction to (1.8), we can obtain

$$
u_{2}=2, u_{5}=34, u_{3 n+8}=18 u_{3 n+5}-u_{3 n+2}, n \in \mathbb{N} \cup\{0\} .
$$

By (1.8), (2.20)-(2.22) and the uniqueness, we immediately arrive at

$$
S_{n}=\frac{1}{2} u_{3 n+2}
$$

and

$$
\begin{aligned}
T_{n} & =\frac{1}{8}\left(u_{3 n+5}-9 u_{3 n+2}\right) \\
& =\frac{1}{8}\left(9 u_{3 n+2}-u_{3 n-1}\right) \\
& =\frac{1}{8}\left[9 u_{3 n+2}+\left(u_{3 n+1}-3 u_{3 n}\right)\right] \\
& =\frac{1}{8}\left[9 u_{3 n+2}+u_{3 n+1}-3\left(3 u_{3 n+1}-u_{3 n+2}\right)\right] \\
& =\frac{3}{2} u_{3 n+2}-u_{3 n+1} .
\end{aligned}
$$

Therefore, we obtain (2.18) and complete the proof of Lemma 2.2.

By Lemma 2.2, we conclude that (2.17) is solvable in integers if and only if $m \mid \frac{1}{2} u_{3 n+2}$ for some $n \in \mathbb{N} \cup\{0\}$. Now we need the following claim:

Claim 1. For $i<j, \frac{1}{2} u_{3 i+2}$ and $\frac{1}{2} u_{3 j+2}$ are relatively prime, i.e.,

$$
\text { g.c.d. }\left(\frac{1}{2} u_{3 i+2}, \frac{1}{2} u_{3 j+2}\right)=1 \text {. }
$$

Proof. By (1.8) one may check that

$$
\begin{aligned}
\text { g.c.d. }\left(u_{3 n+2}, u_{3 n+5}\right) & =\text { g.c.d. }\left(u_{3 n+2}, 8 u_{3 n+3}-3 u_{3 n+2}\right) \\
& =\text { g.c.d. }\left(u_{3 n+2}, 8 u_{3 n+3}\right)=2 .
\end{aligned}
$$

Here we have used facts that g.c.d. $\left(u_{3 n+2}, u_{3 n+3}\right)=1$ and $u_{3 n+2} \equiv 2(\bmod 4)$. As a consequence, for $n \in \mathbb{N} \cup\{0\}, \frac{1}{2} u_{3 n+2}$ and $\frac{1}{2} u_{3 n+5}$ are relatively prime. Hence, by making good use of (2.22) and (2.24), we may apply the mathematical induction to g.c.d. $\left(\frac{1}{2} u_{3 i+2}, \frac{1}{2} u_{3 j+2}\right)$ and get (2.23). 
By Claim 1, for each $n \in \mathbb{N}$ we can choose $m_{n}>1$ satisfying $m_{n} \mid \frac{1}{2} u_{3 n+2}$. Then all $m_{n}$ 's are distinct. This shows that there are infinitely many $m$ 's such that $x^{2}-5 m^{2} y^{2}=-1$ is solvable in integers $x$ and $y$.

On the other hand, if $m$ is not a divisor of $\frac{1}{2} u_{3 n+2}$ for all $n \in \mathbb{N} \cup\{0\}$, then (2.17) is unsolvable in integers. Along with Theorem A1, we immediately obtain that the period of the simple continued fraction of $m \sqrt{5}$ is even.

Therefore, we prove (ii) and complete the proof of Theorem 1.2.

\section{Applications}

For the importance of Theorems 1.1 and 1.2, we introduce some solvable and unsolvable negative Pell equations which can be obtained from Theorems 1.1 and 1.2.

\subsection{Constructing solvable negative Pell equations}

Applying mathematical computations to formulas provided in Theorems 1.1, we can obtain much more solvable negative Pell equations with "fourth-degree-polynomials" coefficients. Here are some examples:

Example 1. Using $u_{0}=u_{1}=1, u_{2}=2, u_{3}=5$ and $u_{4}=13$, by Theorem 1.1 we immediately create the following negative Pell equations which are solvable in integers $x$ and $y$ :

(i) $x^{2}-\left(2 m^{2}-2 m+1\right)\left(2 m^{2}+2 m+1\right) y^{2}=-1$, for $m \in \mathbb{Z}-\{0\}$, has an integer solution $(x, y)=\left(2 m^{2}, 1\right)$; see also, Table 1 .

(ii) $x^{2}-\left(10 m^{2}-6 m+1\right)\left(10 m^{2}+14 m+5\right) y^{2}=-1$, for $m \in \mathbb{Z}$, has an integer solution $(x, y)=$ $\left(10 m^{2}+4 m-2,1\right)$; see also, Table 2 .

(iii) $x^{2}-\left(130 m^{2}+146 m+41\right)\left(130 m^{2}+166 m+53\right) y^{2}=-1$, for $m \in \mathbb{Z}$, has an integer solution $(x, y)=\left(1690 m^{2}+2028 m+606,13\right)$; see also, Table 3 .

(iv) $x^{2}-\left(130 m^{2}+94 m+17\right)\left(130 m^{2}+146 m+41\right) y^{2}=-1$, for $m \in \mathbb{Z}$, has an integer solution $(x, y)=\left(650 m^{2}+600 m+132,5\right)$; see Table 4 for some examples.

Example 2. In Theorem 1.1(i) we can use $u_{10}=4181=37 \cdot 113, u_{11}=10946$ and $u_{12}=28657$ and choose $n=3$ and $m=112$ to get a negative Pell equation

$$
x^{2}-32617 \cdot 33065 y^{2}=-1
$$

which has an integer solution $(x, y)=(179734612,5473)$. Using other methods, such as calculating the length for the period of the simple continued fraction of $\sqrt{32617 \cdot 33065}$ or checking the existence of a primitive Pythagorean triple for $32617 \cdot 33065$, it seems not easy to see that this equation is solvable in integers. 


\begin{tabular}{cc} 
Table $1: x^{2}-\left(2 m^{2}-2 m+1\right)\left(2 m^{2}+2 m+1\right) y^{2}=-1$ \\
\hline \hline $\boldsymbol{m}$ & The corresponding negative Pell equation \\
\hline $\mathbf{1}$ & $x^{2}-5 y^{2}=-1$ \\
$\mathbf{2}$ & $x^{2}-5 \cdot 13 y^{2}=-1$ \\
$\mathbf{3}$ & $x^{2}-5^{2} \cdot 13 y^{2}=-1$ \\
$\mathbf{4}$ & $x^{2}-5^{2} \cdot 41 y^{2}=-1$ \\
$\mathbf{5}$ & $x^{2}-41 \cdot 61 y^{2}=-1$ \\
$\mathbf{6}$ & $x^{2}-5 \cdot 17 \cdot 61 y^{2}=-1$ \\
$\mathbf{7}$ & $x^{2}-5 \cdot 17 \cdot 113 y^{2}=-1$ \\
$\mathbf{8}$ & $x^{2}-5 \cdot 29 \cdot 113 y^{2}=-1$ \\
$\mathbf{9}$ & $x^{2}-5 \cdot 29 \cdot 181 y^{2}=-1$ \\
$\mathbf{1 0}$ & $x^{2}-13 \cdot 17 \cdot 181 y^{2}=-1$ \\
$\mathbf{1 1}$ & $x^{2}-5 \cdot 13 \cdot 17 \cdot 53 y^{2}=-1$ \\
$\mathbf{1 2}$ & $x^{2}-5 \cdot 53 \cdot 313 y^{2}=-1$ \\
$\mathbf{1 3}$ & $x^{2}-5 \cdot 73 \cdot 313 y^{2}=-1$ \\
$\mathbf{1 4}$ & $x^{2}-5 \cdot 73 \cdot 421 y^{2}=-1$ \\
$\mathbf{1 5}$ & $x^{2}-13 \cdot 37 \cdot 421 y^{2}=-1$ \\
$\mathbf{1 6}$ & $x^{2}-5 \cdot 13 \cdot 37 \cdot 109 y^{2}=-1$ \\
$\mathbf{1 7}$ & $x^{2}-5 \cdot 109 \cdot 613 y^{2}=-1$ \\
$\mathbf{1 8}$ & $x^{2}-5 \cdot 137 \cdot 613 y^{2}=-1$ \\
$\mathbf{1 9}$ & $x^{2}-5 \cdot 137 \cdot 761 y^{2}=-1$ \\
$\mathbf{2 0}$ & $x^{2}-292 \cdot 761 y^{2}=-1$ \\
$\mathbf{1}$ &
\end{tabular}




\begin{tabular}{cc} 
Table $2: x^{2}-\left(10 m^{2}-6 m+1\right)\left(10 m^{2}+14 m+5\right) y^{2}=$ \\
\hline \hline $\boldsymbol{m}$ & The corresponding negative Pell equation \\
\hline $\mathbf{- 1 0}$ & $x^{2}-5 \cdot 173 \cdot 1061 y^{2}=-1$ \\
$\mathbf{- 9}$ & $x^{2}-5 \cdot 13 \cdot 53 \cdot 173 y^{2}=-1$ \\
$\mathbf{- 8}$ & $x^{2}-13^{2} \cdot 41 \cdot 53 y^{2}=-1$ \\
$\mathbf{- 7}$ & $x^{2}-13 \cdot 41 \cdot 397 y^{2}=-1$ \\
$\mathbf{- 6}$ & $x^{2}-281 \cdot 397 y^{2}=-1$ \\
$\mathbf{- 5}$ & $x^{2}-5 \cdot 37 \cdot 281 y^{2}=-1$ \\
$\mathbf{- 4}$ & $x^{2}-5 \cdot 37 \cdot 109 y^{2}=-1$ \\
$\mathbf{- 3}$ & $x^{2}-53 \cdot 109 y^{2}=-1$ \\
$\mathbf{- 2}$ & $x^{2}-17 \cdot 53 y^{2}=-1$ \\
$\mathbf{- 1}$ & $x^{2}-17 y^{2}=-1$ \\
$\mathbf{0}$ & $x^{2}-5 y^{2}=-1$ \\
$\mathbf{1}$ & $x^{2}-5 \cdot 29 y^{2}=-1$ \\
$\mathbf{2}$ & $x^{2}-29 \cdot 73 y^{2}=-1$ \\
$\mathbf{3}$ & $x^{2}-73 \cdot 137 y^{2}=-1$ \\
$\mathbf{4}$ & $x^{2}-13 \cdot 17 \cdot 137 y^{2}=-1$ \\
$\mathbf{5}$ & $x^{2}-5^{2} \cdot 13^{2} \cdot 17 y^{2}=-1$ \\
$\mathbf{6}$ & $x^{2}-5^{2} \cdot 13 \cdot 449 y^{2}=-1$ \\
$\mathbf{7}$ & $x^{2}-449 \cdot 593 y^{2}=-1$ \\
$\mathbf{8}$ & $x^{2}-593 \cdot 757 y^{2}=-1$ \\
$\mathbf{9}$ & $x^{2}-757 \cdot 941 y^{2}=-1$ \\
$\mathbf{1 0}$ & $x^{2}-5 \cdot 229 \cdot 941 y^{2}=-1$ \\
$\mathbf{9}$ &
\end{tabular}

Table 3: $\underline{x^{2}-\left(130 m^{2}+146 m+41\right)\left(130 m^{2}+166 m+53\right)} y^{2}=-1$

\begin{tabular}{cc}
$\boldsymbol{m}$ & The corresponding negative Pell equation \\
\hline $\mathbf{- 5}$ & $x^{2}-13 \cdot 197 \cdot 2473 y^{2}=-1$ \\
$\mathbf{- 4}$ & $x^{2}-13 \cdot 29 \cdot 53 \cdot 113 y^{2}=-1$ \\
$\mathbf{- 3}$ & $x^{2}-5^{2} \cdot 29 \cdot 773 y^{2}=-1$ \\
$\mathbf{- 2}$ & $x^{2}-241 \cdot 269 y^{2}=-1$ \\
$\mathbf{- 1}$ & $x^{2}-5^{2} \cdot 17 y^{2}=-1$ \\
$\mathbf{0}$ & $x^{2}-41 \cdot 53 y^{2}=-1$ \\
$\mathbf{1}$ & $x^{2}-317 \cdot 349 y^{2}=-1$ \\
$\mathbf{2}$ & $x^{2}-5 \cdot 181 \cdot 853 y^{2}=-1$ \\
$\mathbf{3}$ & $x^{2}-17 \cdot 97 \cdot 1721 y^{2}=-1$ \\
$\mathbf{4}$ & $x^{2}-5 \cdot 541 \cdot 2797 y^{2}=-1$ \\
$\mathbf{5}$ & $x^{2}-4021 \cdot 4133 y^{2}=-1$ \\
\hline
\end{tabular}


Table 4: $\underline{x^{2}-\left(130 m^{2}+94 m+17\right)\left(130 m^{2}+146 m+41\right)} y^{2}=-1$

\begin{tabular}{cc}
\hline \hline $\boldsymbol{m}$ & The corresponding negative Pell equation \\
\hline $\mathbf{- 5}$ & $x^{2}-13 \cdot 197 \cdot 2797 y^{2}=-1$ \\
$\mathbf{- 4}$ & $x^{2}-29 \cdot 53 \cdot 1721 y^{2}=-1$ \\
$\mathbf{- 3}$ & $x^{2}-5 \cdot 181 \cdot 773 y^{2}=-1$ \\
$\mathbf{- 2}$ & $x^{2}-269 \cdot 349 y^{2}=-1$ \\
$\mathbf{- 1}$ & $x^{2}-5^{2} \cdot 53 y^{2}=-1$ \\
$\mathbf{0}$ & $x^{2}-17 \cdot 41 y^{2}=-1$ \\
$\mathbf{1}$ & $x^{2}-241 \cdot 317 y^{2}=-1$ \\
$\mathbf{2}$ & $x^{2}-5^{2} \cdot 29 \cdot 853 y^{2}=-1$ \\
$\mathbf{3}$ & $x^{2}-13 \cdot 17 \cdot 97 \cdot 113 y^{2}=-1$ \\
$\mathbf{4}$ & $x^{2}-5 \cdot 541 \cdot 2473 y^{2}=-1$ \\
$\mathbf{5}$ & $x^{2}-37 \cdot 101 \cdot 4021 y^{2}=-1$ \\
\hline
\end{tabular}

\subsection{Constructing solvable quadratic Diophantine equations}

By (1.5) and Theorem 1.1, we get a class of solvable quadratic Diophantine equations.

Example 3. The following quadratic Diophantine equations are solvable in integers $X$ and $Y$.

$$
\begin{gathered}
X^{2}-\left(\frac{\left(m^{2}-1\right) u_{3 n+2}}{u_{3 n+1}}+3\right) X Y+m^{2} Y^{2}+1=0, \quad m^{2} \equiv 1\left(\bmod u_{3 n+1}\right), \\
X^{2}-\left(\frac{\left(m^{2}-1\right) u_{3 n-1}}{u_{3 n}}+3\right) X Y+m^{2} Y^{2}+1=0, \quad m^{2} \equiv 1\left(\bmod u_{3 n}\right), \\
X^{2}-\left(\frac{\left(4 m^{2}-1\right) u_{3 n+1}}{u_{3 n}}+3\right) X Y+4 m^{2} Y^{2}+1=0, \quad 4 m^{2} \equiv 1\left(\bmod u_{3 n}\right), \\
X^{2}-\left(\frac{\left(4 m^{2}-1\right) u_{3 n}}{u_{3 n+1}}+3\right) X Y+4 m^{2} Y^{2}+1=0, \quad 4 m^{2} \equiv 1\left(\bmod u_{3 n+1}\right) .
\end{gathered}
$$

Example 3 introduces a lot of nontrivial quadratic Diophantine equations which are solvable. Here we give some examples. Setting $n=1$ in (3.1) and using $u_{4}=13$ and $u_{5}=34$ (by (1.8)), one immediately finds that

$$
X^{2}-\frac{34 m^{2}+5}{13} X Y+m^{2} Y^{2}+1=0
$$

is solvable in integers as $m \equiv \pm 1(\bmod 13)$. Moreover, when $m=12$, we get $X^{2}-377 X Y+$ $144 Y^{2}+1=0$ which has an integer solution $(X, Y)=\left(u_{4}, u_{5}\right)=(13,34)$.

On the other hand, setting $n=1$ in (3.4) and using $u_{3}=5$ and $u_{4}=13$, one finds that

$$
X^{2}-\frac{10 m^{2}+17}{13} X Y+m^{2} Y^{2}+1=0
$$

is solvable in integers as $m \equiv \pm 6(\bmod 13)$. In particular, when $m=6$, the above equation become $X^{2}-29 X Y+36 Y^{2}+1=0$, which has an integer solution $(X, Y)=\left(u_{4}, 2 u_{3}\right)=(13,10)$. 


\subsection{Unsolvability and solvability for $x^{2}-k(k+4) m^{2} y^{2}=-1$}

We introduce some examples for $x^{2}-k(k+4) m^{2} y^{2}=-1$ which are unsolvable or solvable in integers.

Example 4. It is known that $x^{2}-p y^{2}=-1$ is solvable in integers for $p$ a prime congruent to 1 modulo 4 . However, there are many such primes $p_{i}$ 's such that $x^{2}-\left(\prod p_{i}\right) y^{2}=-1$ is unsolvable in integers. For example, by Theorem 1.2(i) one immediately finds that $x^{2}-13 \cdot 17 y^{2}=-1$ $(k=13), x^{2}-37 \cdot 41 y^{2}=-1(k=37), x^{2}-5 \cdot 13 \cdot 61 y^{2}=-1(k=61), x^{2}-5 \cdot 17 \cdot 89 y^{2}=-1$ $(k=85), x^{2}-97 \cdot 101 y^{2}=-1(k=97), \ldots$, and $x^{2}-349 \cdot 353 y^{2}=-1(k=349)$ are unsolvable in integers.

We shall stress that proving the unsolvability of $x^{2}-349 \cdot 353 y^{2}=-1$ via other methods, such as checking the even period length of the simple continued fraction of $\sqrt{349 \cdot 353}$ and checking the non-existence of a primitive Pythagorean triple for $349 \cdot 353$, may be a bit of a challenge.

\section{Example 5.}

(i) One may check directly that the sequence $\left\{u_{n}\right\}_{n \in \mathbb{N} \cup\{0\}}$ modulo 29 obeys a "congruence period"

$$
\left\{u_{n}: n \in \mathbb{N} \cup\{0\}\right\} \equiv\left\{u_{i}: i=0,1, \ldots, 6\right\} \equiv \overline{1,1,2,5,13,5,2}(\bmod 29) .
$$

Hence,

$$
29 \nmid \frac{1}{2} u_{3 n+2}, \quad \forall n \in \mathbb{N} \cup\{0\},
$$

and we conclude from Theorem 1.2(ii) that $x^{2}-5 \cdot 29^{2} y^{2}=-1$ is unsolvable in integers.

(ii) One may check directly that the sequence $\left\{u_{n}\right\}_{n \in \mathbb{N} \cup\{0\}}$ modulo 41 obeys a "congruence period"

$$
\begin{aligned}
&\left\{u_{n}: n \in \mathbb{N} \cup\{0\}\right\} \equiv\left\{u_{i}: i=0,1, \ldots, 19\right\} \\
& \equiv 1,1,2,5,13,-7,7,-13,-5,-2,-1,-1,-2,-5,-13,7,-7,13,5,2 \\
&(\bmod 41) .
\end{aligned}
$$

Hence,

$$
41 \mid \frac{1}{2} u_{3 n+2}, \quad \forall n \in \mathbb{N} \cup\{0\},
$$

and we conclude from Theorem 1.2(ii) that $x^{2}-5 \cdot 41^{2} y^{2}=-1$ is unsolvable in integers.

(iii) By a simple calculation, one may check that

$$
\begin{aligned}
& \left\{u_{n}: n \in \mathbb{N} \cup\{0\}\right\} \equiv\left\{u_{i}: i=0,1, \ldots, 37\right\} \\
\equiv & \frac{1,1,2,5,13,-3,15,11,18,6,0,-6,-18,-11,-15,3,-13,-5,-2,-1}{-1,-2,-5,-13,3,-15,-11,-18,-6,0,6,18,11,15,-3,13,5,2}(\bmod 37) .
\end{aligned}
$$

This shows

$$
37 \mid \frac{1}{2} u_{3 \delta(n)+2}, \quad \forall n \in \mathbb{N} \cup\{0\},
$$

where $\delta(n)=38 n+9$. Hence, $x^{2}-5 \cdot 37^{2} y^{2}=-1$ is solvable in integers. 


\section{Acknowledgements}

We would like to thank the referees for comments and suggestions. This work is supported in part by the Ministry of Science and Technology of Taiwan.

\section{References}

[1] Alpern, D. (2018) Generic two integer variable equation solver: $a x^{2}+b x y+c y^{2}+d x+$ $e y+f-0$. Available online: https : / /www. alpertron.com.ar / QUAD. HTM.

[2] Grytchuk, A., Luca, F. \& Wójtowicz, M. (2000) The negative Pell equation and Pythagorean triples, Proc. Japan Acad. Ser. A Math.Sci., 76, 91-94.

[3] Fouvry, E. \& Klüners, J., On the negative Pell equation, Ann. of Math., (2) 172, 2035-2104.

[4] Keskin, R. (2010) Solutions of some quadratics diophantine equations, Comput. Math. Appl., 60, 2225-2230.

[5] Marlewski, A. \& Marzycki, P. (2004) Infinitely many positive solutions of the Diophantine equation $x^{2}-k x y+y^{2}+x=0$, Comput. Math. Appl., 47, 115-121.

[6] Mollin, R. A. (2010) A Note on the Negative Pell Equation, Internat. J. Algebra, 4, 919-922.

[7] Newman, M. (1977) A note on an equation related to the Pell equation, Amer. Math. Monthly, 84, 365-366.

[8] Niven, I., Zuckerman, H. S., \& Montgomery, H. L. (1991) An Introduction to the Theory of Numbers, Fifth Edition, John Wiley \& Sons, Inc., New York.

[9] Sloane, N. J. A. (1964) The On-Line Encyclopedia of Integer Sequences, Available online: http://oeis.org.

[10] Sierpiński, W. (1964) Elementary Theory of Numbers, Polish Scientific Publishers, Warszawa.

[11] Weil, A. (1984) Number Theory: An Approach Through History, From Hammurapi to Legendre, Birkhäuser, Boston, MA. 


\section{Appendix}

For the reader's convenience, we revisit a well-known property for a second-order linear homogeneous recurrence sequence

$$
A u_{n+2}=B u_{n+1}+C u_{n}, \quad \text { for } n \in \mathbb{N} \cup\{0\}
$$

where $A, B$ and $C$ are real numbers and $A \neq 0$.

Proposition 3.1. Let $u_{n}$ satisfy (3.5). Then we have

$$
C u_{n}^{2}+B u_{n} u_{n+1}-A u_{n+1}^{2}=A\left(u_{n} u_{n+2}-u_{n+1}^{2}\right)=\left(-\frac{C}{A}\right)^{n}\left(C u_{0}^{2}+B u_{0} u_{1}-A u_{1}^{2}\right) .
$$

Proof. We rewrite (3.5) in a matrix form

$$
\left[\begin{array}{cc}
0 & 1 \\
\frac{C}{A} & \frac{B}{A}
\end{array}\right]\left[\begin{array}{cc}
u_{n} & u_{n+1} \\
u_{n+1} & u_{n+2}
\end{array}\right]=\left[\begin{array}{ll}
u_{n+1} & u_{n+2} \\
u_{n+2} & u_{n+3}
\end{array}\right] .
$$

Taking the determinant to (3.7), we get $-\frac{C}{A}\left(u_{n} u_{n+2}-u_{n+1}^{2}\right)=u_{n+1} u_{n+3}-u_{n+2}^{2}$, which immediately implies

$$
u_{n} u_{n+2}-u_{n+1}^{2}=\left(-\frac{C}{A}\right)^{n}\left(u_{0} u_{2}-u_{1}^{2}\right), \quad \forall n \in \mathbb{N} \cup\{0\}
$$

By (3.5) and (3.8), we get (3.6) and complete the proof of Proposition 3.1. 